\title{
Connected Rectilinear Graphs on Point Sets
}

\author{
Maarten Löffler ${ }^{1, \star}$ and Elena Mumford ${ }^{2}$ \\ ${ }^{1}$ Dept. Information and Computing Sciences, Utrecht University, The Netherlands \\ lofflerecs.uu.nl \\ ${ }^{2}$ Dept. of Mathematics and Computer Science, TU Eindhoven, The Netherlands \\ e.mumfordatue.nl
}

\begin{abstract}
Given $n$ points in $d$-dimensional space, we would like to connect the points with straight line segments to form a connected graph whose edges use $d$ pairwise perpendicular directions. We prove that there exists at most one such set of directions. For $d=2$ we present an algorithm for computing these directions (if they exist) in $O\left(n^{2}\right)$ time.
\end{abstract}

\section{Introduction}

Given a set $V$ of $n$ points in $d$-dimensional space, we would like to connect the points of $V$ with straight line segments to form a connected rectilinear graph $G$. A rectilinear graph is an embedded straight-line graph such that any two edges in the graph are either parallel or perpendicular. We define the orientation of such a graph as the set of $d$ pairwise perpendicular directions used by its edges. Two orientations are said to be different if there is a direction $e$ in one of them and a direction $e^{\prime}$ in the other such that $e$ and $e^{\prime}$ are neither the same nor perpendicular. We say that an orientation $O$ allows for a connected rectilinear graph on $V$ if there exists such a graph $G$ that uses $V$ as its vertices and has orientation $O$.

At the Canadian Conference on Computational Geometry in 2007, Therese Biedl asked whether a given set of points in the plane can be the vertex set of two rectilinear polygons that have different orientations. In this paper we show that the answer is no, and more generally, for a set $V$ of points in $\mathbb{R}^{d}$ there exists at most one orientation that allows for a connected rectilinear graph on $V$. Figure 1 shows an example of two rectilinear graphs on the same point set, but note that $G^{\prime}$ is not connected.
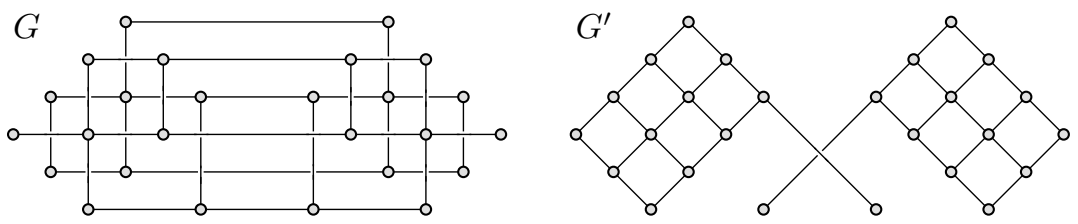

Fig. 1. Two rectilinear graphs with the same vertex set, but different orientations

\footnotetext{
* Supported by the Netherlands Organisation for Scientific Research (NWO) through the project GOGO.
} 
A special case of this problem has been considered by Fekete and Woeginger [4]. They show that for a set of points that have rational coordinates in the plane at most one orientation is possible. Problems on rectilinear polygons have been studied extensively. O'Rourke [10] proves that there is at most one way to connect a given point set into a rectilinear polygon that makes a $90^{\circ}$ turn at each vertex, and gives a simple algorithm to compute it. On the other hand, if turns of $180^{\circ}$ are allowed, Rappaport [12] shows that the problem is NP-hard. Durocher and Kirkpatrick [1] study the problem of finding a collection of rectilinear tours that use the given points as vertices, where the tours are allowed to have different orientations. They prove that this is NP-hard as well.

A number of papers address the problem of drawing a graph on a fixed point set. For example, Pach and Wenger [11] show that to make such a graph planar, a linear number of bends per edge may be necessary. Efrat et al. [3] study the possibility of drawing a crossing-free graph with circular arcs as edges. Rectilinear graphs also received a lot of attention from a graph drawing perspective. Vijayan and Wigderson [13] show how to embed an abstract graph with an additional "direction" associated to each edge as a rectilinear graph in $O\left(n^{2}\right)$ time; Hoffman and Kriegel [7] improve this to $O(n)$ time. Garg and Tamassia [6] show that without such associated directions, it is NP-hard to decide if a graph has a rectilinear embedding.

The remainder of the paper is organised as follows. In Sec. 2 we show that any point set allows for a connected rectilinear graph in at most one orientation. Then, in Sec. 3 we discuss the related algorithmic question of finding such an orientation for a point set in the plane. We conclude in Sec. 4

\section{Existence of Orientations}

In this section, we prove that a point set cannot be the vertex set of two differently oriented rectilinear graphs. We first study the situation in the plane, then we extend the result to any dimension. We use several algebraic concepts, which we try to define briefly when we use them, but we refer to the full version [9] for a more complete and formal discussion.

\subsection{Points in the Plane}

Let $V$ be a set of points in the plane, and let $X$ and $Y$ be the sets of all $x$-coordinates and $y$-coordinates of the vertices in $V$ respectively. Assume w.l.o.g. that $\min (X)=$ $\min (Y)=0$. In this section for convenience we are going to refer to an orientation using the slope on one of its directions since the orientation is uniquely defined by it. Suppose for a contradiction that there are two connected differently oriented rectilinear graphs $G$ and $G^{\prime}$ on $V$. Assume w.1.o.g. that the edges of $G$ are axis-aligned and $G^{\prime}$ has edges of slopes $s$ and $-\frac{1}{s}$.

Let $\mathbb{Q}(s)$ be the field generated by adjoining $s$ to $\mathbb{Q}$; that is, the smallest subfield of $\mathbb{R}$ that contains both $\mathbb{Q}$ and $s$. Consider the vector space $\mathbb{Q}(s)\langle X \cup Y\rangle$; that is, the set of all sums of products of an element from $\mathbb{Q}(s)$ and an element from $X$ or $Y$. Let $E=\left(e_{1}, \ldots, e_{k}\right)$ be a basis for this vector space. We can now denote this vector space by $\mathbb{Q}(s)\langle E\rangle=\mathbb{Q}(s)\left\langle e_{1}, \ldots, e_{k}\right\rangle$. We now have $X, Y \subset \mathbb{Q}(s)\langle E\rangle$ so we can 
write $x_{i}=\sum_{j} x_{i j} e_{j}$ for all $x_{i} \in X$, and $y_{i}=\sum_{j} y_{i j} e_{j}$ for all $y_{i} \in Y$, where $x_{i j}, y_{i j} \in \mathbb{Q}(s)$. We use $[\mathbb{Q}(s): \mathbb{Q}]$ to denote the degree of the extension of field $\mathbb{Q}(s)$ over $\mathbb{Q}$, which is defined as the dimension of $\mathbb{Q}(s)$ as a vector space over $\mathbb{Q}$. We consider the following cases:

$[\mathbb{Q}(s): \mathbb{Q}]=1: s$ is rational.

$[\mathbb{Q}(s): \mathbb{Q}]<\infty: s$ is algebraic over $\mathbb{Q}$.

$[\mathbb{Q}(s): \mathbb{Q}]=\infty: s$ is transcendental over $\mathbb{Q}$.

In fact, the rational case follows directly from the algebraic case since rational numbers are also algebraic, but we have separated them to allow the reader to follow the main argument without needing too much algebraic machinery yet.

Rational Slopes. When $s$ is rational, $\mathbb{Q}(s)=\mathbb{Q}$, therefore $x_{i j}, y_{i j} \in \mathbb{Q}$. We can assume w.l.o.g. that $x_{i j}, y_{i j} \in \mathbb{Z}$ and that their greatest common divisor $(G C D)$ is 1 (if not, scale the input by the appropriate factor). Now $x_{i}, y_{i}$ are elements of the $\mathbb{Z}$-module $\mathbb{Z}\langle E\rangle$; that is, the set of all sums of integer multiples of elements from $E$. Consider any pair of vertices $v, v^{\prime} \in V$, and the horizontal and vertical distances $\Delta x$ and $\Delta y$ between them. These vertices are connected by a path $v_{1}, v_{2}, \ldots, v_{m}$ in $G$, where $v_{1}=v$ and $v_{m}=v^{\prime}$. Denote by $\left(\Delta x_{i}, \Delta y_{i}\right)$ the horizontal and vertical distance between $v_{i}$ and $v_{i+1}$. We know that there exists a path in $G^{\prime}$ from $v_{i}$ to $v_{i+1}$, see Fig. 2. This path uses edges with slope $s$ or $-\frac{1}{s}$, so when following this path we move over distances $(a, s a)$ or $(s b,-b)$. Since all vertex coordinates are in $\mathbb{Z}\langle E\rangle$, we know that $a, b \in \mathbb{Z}\langle E\rangle$. In total we move from $v_{i}$ to $v_{i+1}$ over a distance $\left(a_{i}+s b_{i}, s a_{i}-b_{i}\right)$ where $a_{i}, b_{i} \in \mathbb{Z}\langle E\rangle$. Since $G$ is axisparallel, every edge between two points $v_{i}$ and $v_{i+1}$ is either horizontal or vertical. If it is horizontal, $\Delta y_{i}=s a_{i}-b_{i}=0$, thus $\Delta x_{i}=a_{i}+s b_{i}=a_{i}+s^{2} a_{i}=\left(1+s^{2}\right) a_{i}$. If it is vertical, then $\Delta x_{i}=a_{i}+s b_{i}=0$, thus $\Delta y_{i}=s a_{i}-b_{i}=-s^{2} b_{i}-b_{i}=-\left(1+s^{2}\right) b_{i}$.

Now write $\Delta x_{i}=\sum_{j} \Delta x_{i j} e_{j}$ and $\Delta y_{i}=\sum_{j} \Delta y_{i j} e_{j}$, and also write $a_{i}=\sum_{j} a_{i j} e_{j}$ and $b_{i}=\sum_{j} b_{i j} e_{j}$. Clearly $\Delta x_{i j}, \Delta y_{i j}, a_{i j}, b_{i j} \in \mathbb{Z}$. Since the elements of $E$ are linearly independent over $\mathbb{Q}$, it follows that $\Delta x_{i j}=\left(1+s^{2}\right) a_{i j}$ for horizontal segments and $\Delta y_{i j}=-\left(1+s^{2}\right) b_{i j}$ for vertical segments for all $i, j$.

Now $s^{2} \in \mathbb{Q}$, so we can write $s^{2}=p / q$ with $p$ and $q$ co-prime. This means that $\Delta x_{i j}=(1+p / q) a_{i j}=(p+q) a_{i j} / q$ or $\Delta y_{i j}=-(p+q) b_{i j} / q$. Since $q$ does not divide $p+q$ (unless it is 1 ), $p+q$ is in $\mathbb{Z}$ and divides $\Delta x_{i j}$ and $\Delta y_{i j}$. Since $\Delta x=\sum_{i, j} \Delta x_{i j} e_{j}$, it follows that $p+q$ divides $\Delta x$, and similarly $\Delta y$. So, any two vertices $v$ and $v^{\prime}$ are a $\mathbb{Z}\langle E\rangle$-multiple of $p+q$ away from each other in both horizontal and vertical direction, which contradicts the fact that all their coordinates had GCD 1.

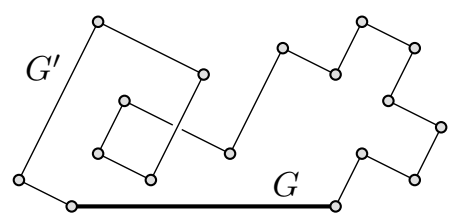

Fig. 2. For any edge of $G$, there is also a path in $G^{\prime}$ connecting its vertices 
Algebraic Slopes. When $s$ is algebraic over $\mathbb{Q}$, the argument described in the previous section still goes through when we replace all instances of $\mathbb{Q}$ by $\mathbb{Q}(s)$ and $\mathbb{Z}$ by $O_{\mathbb{Q}(s)}$. Here $O_{\mathbb{Q}(s)}$ is the ring of integers of $\mathbb{Q}(s)$, which consists of all algebraic integers that are in $\mathbb{Q}(s)$. Intuitively, the ring of integers $O_{\mathbb{Q}(s)}$ behaves towards the field $\mathbb{Q}(s)$ as $\mathbb{Z}$ behaves towards $\mathbb{Q}$. Every element of $\mathbb{Q}(s)$ can be written as $p / q$, where $p, q \in O_{\mathbb{Q}(s)}$. Every element of $O_{\mathbb{Q}(s)}$ can be written as the product of a finite sequence of irreducible elements of $O_{\mathbb{Q}(s)}$, though this factorisation is not necessarily unique. This means we can divide out common divisors in $O_{\mathbb{Q}(s)}$, and that we can have irreducible fractions $p / q$ in $\mathbb{Q}(s)$.

Transcendental Slopes. When $s$ is transcendental, every element $w \in \mathbb{Q}(s)$ can be written in the form

$$
w=\frac{\sum_{0 \leq l \leq h} w_{l} s^{l}}{\sum_{0 \leq l^{\prime} \leq h^{\prime}} w^{\prime} l^{\prime} s^{l^{\prime}}}
$$

for some $h, h^{\prime} \in \mathbb{N}$, and $w_{l}, w^{\prime} l^{\prime} \in \mathbb{Q}$. Assume w.l.o.g. that we can write

$$
x_{i j}=\sum_{0 \leq l \leq h} x_{i j l} s^{l} \text { and } y_{i j}=\sum_{0 \leq l \leq h} y_{i j l} s^{l}
$$

where $h \in \mathbb{N}$ and $x_{i j l}, y_{i j l} \in \mathbb{Z}$ (otherwise scale the input).

Now $x_{i j}, y_{i j} \in \mathbb{Z}\left\langle s, \ldots, s^{h}\right\rangle$. Assume $h \geq 2$ (if it is smaller, just add some 0's to the descriptions of the coordinates). We now also know that $\left(1+s^{2}\right) \in \mathbb{Z}\left\langle s, \ldots, s^{h}\right\rangle$. Assume w.l.o.g. that not all of $x_{i j}, y_{i j}$ can be written as $\left(1+s^{2}\right) w$ for some $w \in$ $\mathbb{Z}\left\langle s, \ldots, s^{h}\right\rangle$ (otherwise divide everything by $\left(1+s^{2}\right)$ ).

However, in the same way as before, we argue that $\Delta x_{i j}=\left(1+s^{2}\right) a_{i j}$ for horizontal segments and $\Delta y_{i j}=-\left(1+s^{2}\right) b_{i j}$ for vertical segments for all $i, j$, where now $a_{i j}, b_{i j} \in \mathbb{Z}\left\langle s, \ldots, s^{h}\right\rangle$. This clearly contradicts our assumption. Thus we arrive at the following theorem:

Theorem 1. Given a set of points in the plane, there can be at most one orientation that allows for a connected rectilinear graph that has these points as its vertices.

\subsection{Points in Higher Dimensions}

Let $V$ be a set of $n$ points in $\mathbb{R}^{d}$. We will show that there is at most one orientation that allows for a connected rectilinear graph that uses $V$ as its vertex set. Suppose for a contradiction there are two connected rectilinear graphs $G$ and $G^{\prime}$ on $V$. And let $E$ be the orientation of $G$ and $E^{\prime}$ be the orientation of $G^{\prime}$. Let $e \in E$ and $e^{\prime} \in E^{\prime}$ be two distinct directions that are not perpendicular.

Let $\alpha$ be a plane spanned by $e$ and $e^{\prime}$. Let $V_{\alpha}$ be the projection of $V$ on $\alpha$, and let $G_{\alpha}$ and $G_{\alpha}^{\prime}$ be the projections of $G$ and $G^{\prime}$ on $\alpha$. We ignore any duplicate points in $V_{\alpha}$ and edges that were reduced to single points in $G_{\alpha}$ and $G_{\alpha}^{\prime}$. Note that $G_{\alpha}$ and $G_{\alpha}^{\prime}$ are still connected graphs. Moreover, since $\alpha$ contains $e$, all edges of $G_{\alpha}$ map either to an edge in $\alpha$ parallel to $e$, or to one perpendicular to $e$, so $G_{\alpha}$ is a rectilinear graph. Similarly, $G_{\alpha}^{\prime}$ is a rectilinear graph. However, these are two graphs on the same vertex set in 2-dimensional space, which is not possible by Theorem 1 We have proven the following theorem: 
Theorem 2. Given a set of points in $\mathbb{R}^{d}$, there exists at most one orientation that allows for a connected rectilinear graph that has these points as its vertices.

\section{Finding the Right Orientation}

We now discuss the algorithmic side of the problem: given a set $V$ of points in the plane, can we find a slope $s$ such that the graph on $V$ with edges of slope $s$ and $-\frac{1}{s}$ is connected? A trivial approach takes $O\left(n^{2} \log n\right)$ time. Consider all pairs of points and the line segment connecting them, and sort those segments by slope. For each slope that has at least $n-1$ segments (together with its perpendicular slope), we can test whether they form a connected graph in linear time. Note that the most expensive step here is sorting the directions: a long-standing open problem is whether this can be done any faster than in $O\left(n^{2} \log n\right)$ time [5].

However, it is not necessary to sort all directions, since many of them are uninteresting. Namely, since our graph has to be connected, an arbitrary point $p$ has to share an edge with at least one other point of $V$. Thus we only need to consider $n-1$ (possibly non-distinct) directions obtained by connecting $p$ to all other points in $V$. Now consider the problem in dual space. Our set of points becomes a set of lines, our slope an $x$-coordinate, and two points are connected by a line segment of slope $s$ if the two corresponding lines intersect at $x$-coordinate $s$. We sweep two vertical lines (at $x=-\frac{1}{s}$ and $x=s$ ) simultaneously over the dual plane, and keep track of the intersection points on those lines. The arrangement of the lines can be computed in $O\left(n^{2}\right)$ time [2]. We can inspect the potentially interesting slopes, and process the events in between in $O\left(n^{2}\right)$ time in total. The details are not hard, and can be found in the full version [9].

Deciding whether there is an orientation that allows for a planar rectilinear graph (a simple polygon, for example) on a given set of points is NP-hard: Since there is at most one possible orientation, we can use the algorithm sketched above to find it. Then we can take the maximal rectilinear graph in that orientation. However, now we need to decide whether this graph has a non-crossing subgraph, which is NP-complete [8].

\section{Conclusion}

We have proven that given a point set in $\mathbb{R}^{d}$, there exists at most one orientation such that the maximal rectilinear graph on the points in that orientation is connected. However, finding this orientation remains an interesting challenge. We have shown that this can be done in $O\left(n^{2}\right)$ time for a 2-dimensional point set, but we see no reason for this bound to be tight. Furthermore, finding such an orientation in higher dimensions is still open. We have also shown that deciding whether the points can be connected into a planar rectilinear graph is NP-hard.

\section{Acknowledgments}

The authors would like to thank Therese Biedl for introducing them to the problem, and Oswin Aichholzer, Chris Gray and Rodrigo Silveira for fruitful discussions on the subject. 


\section{References}

1. Durocher, S., Kirkpatrick, D.: On the hardness of turn-angle-restricted rectilinear cycle cover problems. In: CCCG 2002, pp. 13-16 (2002)

2. Edelsbrunner, H., Guibas, L.: Topologically sweeping an arrangement. J. Comput. Syst. Sci. 38, 165-194 (1989)

3. Efrat, A., Erten, C., Kobourov, S.: Fixed-location circular-arc drawing of planar graphs. In: Liotta, G. (ed.) GD 2003. LNCS, vol. 2912, pp. 147-158. Springer, Heidelberg (2004)

4. Fekete, S., Woeginger, G.: Angle-restricted tours in the plane. Comput. Geom. Theory Appl. 8(4), 195-218 (1997)

5. Fredman, M.: How good is the information theory bound in sorting? Theoret. Comput. Sci. 1, 355-361 (1976)

6. Garg, A., Tamassia, R.: On the computational complexity of upward and rectilinear planarity testing. SIAM J. on Computing 31(2), 601-625 (2002)

7. Hoffman, F., Kriegel, K.: Embedding rectilinear graphs in linear time. Inf. Process. Lett. 29(2), 75-79 (1988)

8. Jansen, K., Woeginger, G.: The complexity of detecting crossingfree configurations in the plane. BIT 33(4), 580-595 (1993)

9. Löffler, M., Mumford, E.: Connected rectilinear polygons on point sets (2008), http://www.cs.uu.nl/research/techreps/UU-CS-2008-028.html

10. O'Rourke, J.: Uniqueness of orthogonal connect-the-dots. In: Toussaint, G. (ed.) Computational Morphology, pp. 97-104 (1988)

11. Pach, J., Wenger, R.: Embedding planar graphs at fixed vertex locations. In: Whitesides, S.H. (ed.) GD 1998. LNCS, vol. 1547, pp. 263-274. Springer, Heidelberg (1999)

12. Rappaport, D.: On the complexity of computing orthogonal polygons from a set of points. Technical Report TR-SOCS-86.9, McGill Univ., Montreal, PQ (1986)

13. Vijayan, G., Wigderson, A.: Rectilinear graphs and their embeddings. SIAM J. on Computing 14(2), 355-372 (1985) 